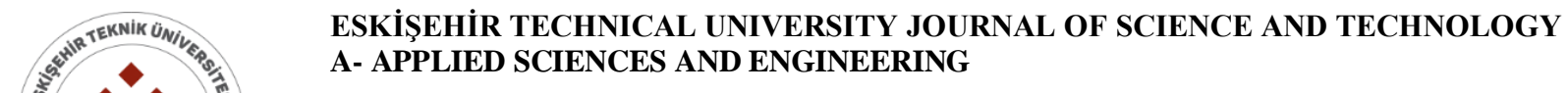

2019, Vol: 20, pp. 170 - 180, DOI: 10.18038/estubtda.649511

\title{
THE STUDY OF CLASSICS PARTICLES' ENERGY AT ARCHIMEDEAN SOLIDS WITH CLIFFORD ALGEBRA
}

\author{
Şadiye ÇAKMAK ${ }^{1}$, Abidin KILIÇ ${ }^{2 *}$ \\ ${ }^{1}$ Eskişehir Osmangazi University, Eskişehir, Turkey \\ ${ }^{2}$ Department of Physics, Faculty of Science, Eskişehir Technical University, Eskişehir, Turkey
}

\begin{abstract}
Geometric algebras known as a generalization of Grassmann algebras complex numbers and quaternions are presented by Clifford (1878). Geometric algebra describing the geometric symmetries of both physical space and spacetime is a strong language for physics. Groups generated from `Clifford numbers` is firstly defined by Lipschitz (1886). They are used for defining rotations in a Euclidean space. In this work, Clifford algebra are identified. Energy of classic particles with Clifford algebra are defined. This calculations are applied to some Archimedean solids. Also, the vertices of Archimedean solids presented in the Cartesian coordinates are calculated.
\end{abstract}

Keywords: Clifford Algebra, Energy of Archimedean solids's, Archimedean solids

\section{INTRODUCTION}

The ideas and concepts of physics are best expressed in the language of mathematics. But this language is far from unique. Many different algebraic systems exist and are in use today, all with their own advantages and disadvantages. In this paper, we describe what we believe to be the most powerful available mathematical system developed to date. This is geometric algebra, which is presented as a new mathematical tool to add to your existing set as either a theoretician or experimentalist. Our aim is to introduce the new techniques via their applications, rather than as purely formal mathematics. These applications are diverse, and throughout we emphasize the unity of the mathematics underpinning each of these topics.

The history of geometric algebra is one of the more unusual tales in the development of mathematical physics. William Kingdom Clifford introduced his geometric algebra in the 1870s, building on the earlier work of Hamilton and Grassmann. It is clear from his writing that Clifford intended his algebra to describe the geometric properties of vectors, planes and higher-dimensional objects. But most physicists first encounter the algebra in the guise of the Pauli and Dirac matrix algebras of quantum theory. Few then contemplate using this unwieldy matrix for practical geometric computing. Indeed, some physicists come away from a study of Dirac theory with the view that Clifford's algebra is inherently quantum-mechanical [1].

Wessel, Argand, and Gauss used the complex numbers in the solutions of two-dimensional problems. The exponential form of complex numbers is useful in the theory of rotational motions. The quaternion algebra, which was defined by Sir W. R. Hamilton, was generalized for the three-dimensional complex numbers [2]. The quaternion algebra is the Clifford algebra of the two-dimensional anti-Euclidean space. Quaternions in the three-dimensional spaces have more useful appearances for the subalgebras of Clifford algebra. In the n-dimensional spaces, Grassmann carried on the studies for the multidimensional bodies and defined the central product, which includes both interior and exterior products. Grassmann's central product is the Clifford product of vectors. Clifford tried to combine Grassmann's

*Corresponding Author:abkilic@eskisehir.edu.tr

Received: 13.10.2019 Published: 16.12.2019 
algebra and quaternions in a mathematical system. Then this study, which was entitled "Application of Grassmann's Extensive Algebra", was published [3].

Today, Clifford algebra has an important role in the investigations of the symmetry properties of systems, crystallography, molecular and solid-state physics.

The method of point groups in the multi-dimensional spaces is derived by transforming into the parameters of the reflections and possible rotational operations. In the three-dimensional spaces, Altmann showed that the Euler's angles are not useful for the rotational operations but Euler-Rodriques' parameters are more advantageous [4]. To know the rotational pole and angle for each rotational operations is necessary and enough. So, there is a similarity between proper and improper operations in.

The rest of the paper is organized within 4 sections. Section 2 reveals the quaternions and symmetry operations in. In section 3, the Clifford algebra and symmetry operations are shown. An application of the symmetry operations with Clifford algebra is given in section 4. Conclusions are drawn in the last section [5].

\section{QUATERNIONS AND SYMMETRY OPERATIONS IN R $\mathbf{R}^{3}$}

The abstract quaternion group, discovered by William Rowan Hamilton in 1843, is an illustration of group structure [6]. A quaternion is a quantity represented symbolically by $\mathbf{A}$ and it is defined through the following equations

$$
\mathbf{A}=\mathrm{a} 1+A_{x} \mathbf{i}+A_{y} \mathbf{j}+A_{z} \mathbf{k}
$$

or $\mathbf{A}=[\mathrm{a}, \overrightarrow{\mathrm{A}}], \quad \overrightarrow{\mathrm{A}}=\left(\mathbf{A}_{\mathbf{x}}, \mathbf{A}_{\mathbf{y}}, \mathbf{A}_{\mathbf{z}}\right), \quad$ where all a, $A_{x}, A_{y}, A_{z}$ coefficients are the real numbers. The unitary quaternions $\mathbf{i}, \mathbf{j}, \mathbf{k}$ satisfy the multiplication rules as follows:

$$
\mathbf{i} \mathbf{j}=\mathbf{k}, \quad \mathbf{j} \mathbf{i}=-\mathbf{k}, \mathbf{j} \mathbf{k}=\mathbf{i}, \quad \mathbf{k} \mathbf{j}=-\mathbf{i}, \quad \mathbf{i} \mathbf{k}=\mathbf{- j}, \quad \mathbf{k} \mathbf{i}=\mathbf{j} .
$$

Also $\mathbf{i}, \mathbf{j}$ and $\mathbf{k}$ can be written as $\mathbf{e}_{1}, \boldsymbol{e}_{2}$ and $\mathbf{e}_{3}$, reciprocally. The vector quaternion $\mathbf{A}$ with components $\left[0, A_{x}, A_{y}, A_{z}\right]$ and a vector $\overrightarrow{\mathrm{A}}$ of the Euclidean tridimensional space with components $\left(A_{x}, A_{y}, A_{z}\right)$ are reciprocally associated [7].

If $\mathrm{A}$ and $\mathrm{B}$ quaternions are

$$
A=a 1+A_{x} e_{1}+A_{y} e_{2}+A_{z} e_{3}=\left[a, \vec{A}_{]},\right.
$$

and

$$
\mathrm{B}=\mathrm{b} 1+\mathrm{B}_{\mathrm{xe} 1}+\mathrm{B}_{\mathrm{y}} \mathrm{e}_{2}+\mathrm{B}_{\mathrm{z}} \mathrm{e}_{3}=[\mathrm{b}, \overrightarrow{\mathrm{B}}]
$$

the product of two quaternions, namely $A$ and $B$, is given by $A B=\left[a b-\vec{A} \cdot \vec{B}, a{ }^{3}+b \vec{A}+\vec{A} \times \vec{B}\right.$,

$$
\begin{aligned}
& A B=\left(a b-A_{x} B_{x}-A_{y} B_{y}-A_{z} B_{z}\right)+e_{1}\left(A_{x} b+a B_{x}+A_{y} B_{z}-A_{z} B_{y}\right)+ \\
& e_{2}\left(a B_{y}+A_{y} b-A_{x} B_{z}+A_{z} B_{x}\right)+e_{3}\left(a_{z}+A_{z} b+A_{x} B_{y}-A_{y} B_{x}\right),
\end{aligned}
$$


where the result is a quaternion. It must be noted that the product of quaternions is not commutative, but associative. The product of $\mathbf{A}$ and $\mathbf{B}$ quaternions in the matrix form can be written.

For each quaternion $\mathrm{A}$, its conjugate is [8]

$$
A^{*}=a l-A_{x} e_{1}-A_{y} e_{2}-A_{z} e_{3} .
$$

The rotation of arbitrary points on an unit sphere $(\mathbf{R}(\gamma \mathbf{k}))$ can be defined by $\gamma$ angle around of the definite k-axis $(0<\gamma<\Pi)$. The poles of rotational operations are defined on the every half-sphere. In general, the rotations (counterclokwise) are accepted to be positive direction. If $\mathbf{R}(\beta \mathbf{l})$ and $\mathbf{R}(\alpha \mathbf{m})$ are the rotations with 8 and $\alpha$ angles around of 1 and $m$ axes, respectively, then the result of two rotational operations has to be equal to a new rotation with $\gamma$-angle around of $\mathrm{k}$ - axis, i.e.

$$
\mathbf{R}(\beta \mathbf{l}) \mathbf{R}(\alpha \mathbf{m})=\mathbf{R}(\gamma \mathbf{k})
$$

where $\mathrm{k}, 1, \mathrm{~m}$ are the axial vectors that correspond to the $\mathrm{k}, 1$, $\mathrm{m}$ rotation axes, respectively. Rodriques [9] defined the geometrical structure and algebra for the angles $\gamma, \beta, \alpha$ and the axes $\mathrm{k}, 1, \mathrm{~m}$ in the following forms

$$
\begin{aligned}
\cos \frac{\gamma}{2} & =\cos \frac{\alpha}{2} \cos \frac{\beta}{2}-\sin \frac{\alpha}{2} \sin \frac{\beta}{2} \mathbf{m} \cdot \mathrm{l}, \\
\sin \frac{\gamma}{2} k & =\sin \frac{\alpha}{2} \cos \frac{\beta}{2} \mathrm{~m}-\cos \frac{\alpha}{2} \sin \frac{\beta}{2} \mathrm{l}+\sin \frac{\alpha}{2} \sin \frac{\beta}{2} \mathrm{~m} \times \mathrm{l} .
\end{aligned}
$$

The rotational operations with the normalized quaternions can be written as

$$
\begin{aligned}
& \mathbf{R}(\alpha \mathbf{m})=\left[\cos \frac{\alpha}{2}, \sin \frac{\alpha}{2} \mathbf{m}\right], \\
& \mathbf{R}(\beta \mathbf{l})=\left[\cos \frac{\beta}{2}, \sin \frac{\beta}{2} \mathbf{l}\right] .
\end{aligned}
$$

In the similar way, from Eqs. (7), (8), (9), $\mathbf{R}(\gamma \mathbf{k})$ can be defined by the following equations:

$$
\begin{aligned}
& \mathbf{R}(\gamma \mathbf{k})=\left[\cos \frac{\alpha}{2}, \sin \frac{\alpha}{2} \mathbf{m}\right]\left[\cos \frac{\beta}{2}, \sin \frac{\beta}{2} \mathbf{l}\right], \\
& \mathbf{R}(\gamma \mathbf{k})=\left[\cos \frac{\gamma}{2}, \sin \frac{\gamma}{2} \mathbf{k}\right] .
\end{aligned}
$$

Any point represented by $\mathbf{R}$ quaternion transforms to the a new quaternionic point at the end of a rotation defined by $\mathbf{A}$ unit quaternion. This new quaternion is;

$$
\mathrm{R}^{\prime}=\mathbf{A} \mathbf{R} \mathbf{A}^{*}
$$

where $\mathrm{A}^{*}$ is complex conjugate of $\mathbf{A}[5]$.

\section{SYMMETRY OPERATIONS WITH CLIFFORD ALGEBRA}

Clifford algebra is used for the studies of symmetry in physics. There are three basic units $\mathrm{e}_{\mathrm{i}}(i=1,2$, 3) in Clifford algebra such that 


$$
\mathbf{e}_{\mathrm{i}} \mathbf{e}_{\mathbf{j}}+\mathbf{e}_{\mathbf{j}} \mathbf{e}_{\mathrm{i}}=\mathbf{2} \boldsymbol{\delta}_{\mathrm{ij}}
$$

which are equivalent to

$\mathrm{e}_{\mathrm{i}} \mathrm{e}_{\mathrm{j}}=1,(16)$

$$
e_{i} e_{j}=-e_{j} e_{i}
$$

The easiest way to understand the geometric product is by example, so consider a two-dimensional space (a plane) spanned by two orthonormal vectors $\mathbf{e}_{\mathbf{1}}$ and $\mathbf{e}_{2}$. These basis vectors satisfy

$$
\begin{aligned}
& \mathrm{e}_{1}^{2}=\mathrm{e}_{2}^{2}=1 \\
& \mathrm{e}_{1} \cdot \mathrm{e}_{2}=0
\end{aligned}
$$

The final entity present in the algebra is the bi-vector $\mathrm{e}_{1} \Lambda \mathrm{e}_{2}$. This is the highest grade element in the algebra, since the outer product of a set of dependent vectors is always zero. The Clifford product of two vectors, namely $\vec{a}$ and $\vec{b}$, with components

$$
\overrightarrow{\mathrm{a}}=\mathrm{a}_{1} \mathrm{e}_{1}+\mathrm{a}_{2} \mathrm{e}_{2},
$$

and

$$
\overrightarrow{\mathrm{b}}=\mathrm{b}_{1} \mathrm{e}_{1}+\mathrm{b}_{2} \mathrm{e}_{2}
$$

is given by

$$
\vec{a} \vec{b}=\vec{a} \cdot \vec{b}+\vec{a} \Lambda \vec{b}=a_{1} b_{1}+a_{2} b_{2}+\left(a_{1} b_{2}-a_{2} b_{1}\right) \mathbf{e}_{12},
$$

where " $\Lambda$ " is called as the wedge product [10]. To study the properties of the bivec- tor $\mathrm{e}_{1} \Lambda \mathrm{e}_{2}$ we first recall that for orthogonal vectors the geometric product is a pure bivector:

$$
\mathrm{e}_{1} \mathrm{e}_{2}=\mathrm{e}_{1} \cdot \mathrm{e}_{2}+\mathrm{e}_{1} \Lambda \mathrm{e}_{2}=\mathrm{e}_{1} \Lambda \mathrm{e}_{2}=\mathrm{e}_{12}
$$

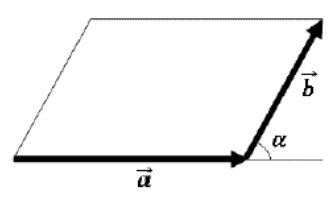

Figure 1. The geometrical meaning of a $\Lambda \mathrm{b}[11]$.

and that orthogonal vectors anticommute:

$$
\mathrm{e}_{2} \mathrm{e}_{1}=\mathrm{e}_{2} \Lambda \mathrm{e}_{1}=-\mathrm{e}_{1} \Lambda \mathrm{e}_{2}=-\mathrm{e}_{1} \mathrm{e}_{2}
$$

We can now form products in which $\mathrm{e}_{2} \mathrm{e}_{1}$ multiplies vectors from the left and the right. First from the left we find that

and

$$
\left(\mathrm{e}_{1} \Lambda \mathrm{e}_{2}\right) \mathrm{e}_{1}=\left(-\mathrm{e}_{2} \mathrm{e}_{1}\right) \mathrm{e}_{1}=-\mathrm{e}_{2} \mathrm{e}_{1} \mathrm{e}_{1}=-\mathrm{e}_{2}
$$

$$
\left(\mathrm{e}_{1} \Lambda \mathrm{e}_{2}\right) \mathrm{e}_{2}=\left(\mathrm{e}_{1} \mathrm{e}_{2}\right) \mathrm{e}_{2}=\mathrm{e}_{1} \mathrm{e}_{2} \mathrm{e}_{2}=\mathrm{e}_{1}
$$


If we assume that $e_{1}$ and $e_{2}$ form a right-handed pair, we see that left multiplication by the bivector rotates vectors $90^{\circ}$ clockwise(i.e. in a negative sense).

Similarly, acting from the right

$$
\begin{aligned}
& \mathrm{e}_{1}\left(\mathrm{e}_{1} \mathrm{e}_{2}\right)=\mathrm{e}_{2}, \\
& \mathrm{e}_{2}\left(\mathrm{e}_{1} \mathrm{e}_{2}\right)=-\mathrm{e}_{1},
\end{aligned}
$$

So right multiplication rotates $90^{\circ}$ anticlockwise -a positive sense.

The final product in the algebra to consider is the square of the bivector $\mathrm{e}_{1} \Lambda \mathrm{e}_{2}$ :

$$
\left(e_{1} \Lambda e_{2}\right)^{2}=e_{1} e_{2} e_{1} e_{2}=-e_{1} e_{1} e_{2} e_{2}=-1 .
$$

Geometric consideration have led naturally to quantity which square to -1 . This fits with the fact that two successive left (or right) multiplication of a vector by $\mathrm{e} \mathrm{e}_{2}$ rotates the vector through $180^{\circ}$, which is equivalent to multiplying by $-1.1, \mathrm{e}_{1}, \mathrm{e}_{2}, \mathrm{e}_{12}$ form the basis of the Clifford algebra $C l_{2}$ of the vector plane $R^{2}$.

The Clifford algebra $\mathrm{Cl}_{2}$ is the four-dimensional linear space and its basis elements have the multiplication table as follows:

Table 1. The basis of Clifford Algebra

\begin{tabular}{c|ccc} 
& $\mathrm{e}_{1}$ & $\mathrm{e}_{2}$ & $\mathrm{e}_{12}$ \\
\hline $\mathrm{e}_{1}$ & 1 & $\mathrm{e}_{12}$ & $\mathrm{e}_{2}$ \\
$\mathrm{e}_{2}$ & $-\mathrm{e}_{12}$ & 1 & $-\mathrm{e}_{1}$ \\
$\mathrm{e}_{12}$ & $-\mathrm{e}_{2}$ & $\mathrm{e}_{1}$ & -1
\end{tabular}

The reflection of $\vec{r}$ across the line $\vec{a}$, namely the mirror image $\vec{r}$ of $\vec{r}$ with respect to $\overrightarrow{\mathrm{a}}$ is given by

$$
\overrightarrow{\mathrm{r}}=\overrightarrow{\mathrm{a}} \overrightarrow{\mathrm{r}} \overrightarrow{\mathrm{a}}^{-1}
$$

Equation (30) can be directly obtained from using the commutation properties of Clifford algebra [10]. The composition of two reflections,

first across $\vec{a}$ and then across $\vec{b}$, is given by

$$
\overrightarrow{\mathrm{r}}=\overrightarrow{\mathrm{b}} \overrightarrow{\mathrm{r}} \overrightarrow{\mathrm{b}}^{-1}=\overrightarrow{\mathrm{b}}\left(\overrightarrow{\mathrm{a}} \overrightarrow{\mathrm{r}} \overrightarrow{\mathrm{a}}^{-1}\right) \overrightarrow{\mathrm{b}}^{-1}=(\overrightarrow{\mathrm{b}} \overrightarrow{\mathrm{a}}) \overrightarrow{\mathrm{r}}(\overrightarrow{\mathrm{b}} \overrightarrow{\mathrm{a}})^{-1}
$$

The composite of these two reflections is a rotation by twice the angle between $\vec{a}$ and $\vec{b}$. 


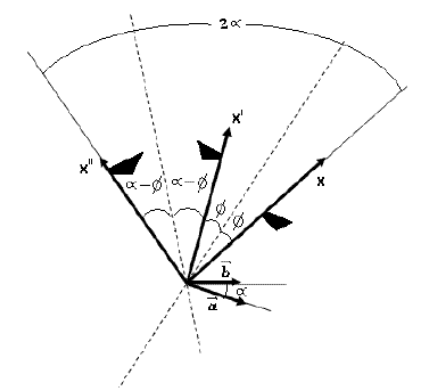

Figure 2. When $\mathbf{x}$ is subjected two successive reflection first with respect to a plane perpendicular to a and then with to a plane perpendicular to $b$, the result is a rotation of $\mathbf{x}$ about an axis in the direction axb.

1 the scalar
$\mathrm{e}_{1}, \mathrm{e}_{2}, \mathrm{e}_{3}$
Vectors
$\mathrm{e}_{1} \mathrm{e}_{2}, \mathrm{e}_{1} \mathrm{e}_{3}, \mathrm{e}_{2} \mathrm{e}_{3}$
bivectors
$\mathrm{e}_{1} \mathrm{e}_{2} \mathrm{e}_{3}$
a volume element

An arbitrary element in $\mathrm{Cl}_{3}$ is a sum of a scalar, a vector, a bivector and a volume element, and can be written as

$$
u=u_{0}+u_{1} e_{1}+u_{2} e_{2}+u_{3} e_{3}+u_{12} e_{12}+u_{13} e_{13}+u_{23} e_{23}+u_{123} e_{123}
$$

The Clifford units, ei 's, are identified with orthogonal reflections (mirrors)

$$
\mathrm{e}_{1} \leftrightarrow \sigma_{y z}, \mathrm{e}_{2} \leftrightarrow \sigma_{\mathrm{xz}}, \mathrm{e}_{3} \leftrightarrow \sigma_{\mathrm{xy}}
$$

The mappings between the Clifford bivectors $\mathbf{e}_{\mathbf{i}} \mathbf{e}_{\mathbf{j}}$ and the corresponding quaternion units are defined

$$
\begin{aligned}
& \mathrm{e}_{3} \mathrm{e}_{2} \leftrightarrow \sigma_{\mathrm{xy}} \sigma_{\mathrm{xz}}=\mathrm{C}_{2 \mathrm{x}} \leftrightarrow[0,(1,0,0)], \\
& \mathrm{e}_{1} \mathrm{e}_{3} \leftrightarrow \sigma_{\mathrm{yz}} \sigma_{\mathrm{xy}}=\mathrm{C}_{2 \mathrm{y}} \leftrightarrow[0,(0,1,0)], \\
& \mathrm{e}_{2} \mathrm{e}_{1} \leftrightarrow \sigma_{\mathrm{xz}} \sigma_{\mathrm{yz}}=\mathrm{C}_{2 \mathrm{z}} \leftrightarrow[0,(0,0,1)] .
\end{aligned}
$$

The inversion, which is a product of three reflections, is obtained by a trivector as follows:

$$
\mathrm{e}_{1} \mathrm{e}_{2} \mathrm{e}_{3} \leftrightarrow \sigma_{\mathrm{yz}} \sigma_{\mathrm{xz}} \sigma_{\mathrm{xy}}=\mathrm{i}
$$

where $\mathbf{e}_{1} \mathbf{e}_{2} \mathbf{e}_{3} \in \mathrm{Cl}_{3}$ is performed [5].

\section{AN APPLICATION OF THE SYMMETRY OPERATIONS WITH CLIFFORD ALGEBRA}

The reflection and rotation operations in the solid state physics and molecular physics play an important role.

Table 2. Numbers for the five Platonic solids

\begin{tabular}{|l|c|c|c|c|}
\hline & Number of faces & Number of edges & Number of vertices & Edges per face \\
\hline Tetrahedron & 4 & 6 & 4 & 3 \\
\hline Cube & 6 & 12 & 8 & 4 \\
\hline Octahedron & 8 & 12 & 6 & 3 \\
\hline Icosahedron & 20 & 30 & 12 & 3 \\
\hline Dodecahedron & 12 & 30 & 20 & 5 \\
\hline
\end{tabular}


The symmetry operations can be easily applied on the regular polyhedra, which are called Platonic Solids., and semi-regular poly-hedra, which are called Archimedean Solids. The Platonic solids are tetrahedron, cube, octahedron, icosahedron and dodecahedron. Some important numbers for the Platonic solids are shown in Table 2. The Archimedean solids are truncated tetrahedron, cuboctahedron, truncated cube, truncated octahedron, rhombicuboctahedron (or small rhombicuboctahedron), truncated cuboctahedron (or great rhombicuboctahedron), snub cube ( or snub hexahedron), icosidodecahedron, truncated dodecahedron, truncated icosahedron, rhombicosidodecahedron (or small rhombicosidodecahedron), truncated icosidodecahedron (or great rhombicosidodecahedron), snub dodecahedron (or snub icosidodecahedron). Some important numbers for the Platonic solids are shown in Table 3 [14].

Table 3. Numbers for the thirteen Archimedean solids

\begin{tabular}{|c|c|c|c|}
\hline & Number of faces & Number of edges & Number of \\
\hline Truncated Tetrahedron & $8(4$ triangles, 4 hexagons $)$ & 18 & 12 \\
\hline Cuboctahedron & 14 ( 8 triangles, 6 squares) & 24 & 12 \\
\hline Truncated Cube & 14 ( 8 triangles, 6 octagons) & 36 & 24 \\
\hline Truncated Octahedron & 146 squares, 8 hexagons) & 36 & 24 \\
\hline Rhombicuboctahedron & 26 ( 8 triangles, 18 squares) & 48 & 24 \\
\hline Truncated Cuboctahedron & 26 (12 squares, 8 hexagons, 6 octagons) & 72 & 48 \\
\hline Snub Cube & 38 (32 triangles, 6 squares) & 60 & 24 \\
\hline Icosidodecahedron & 32 (20 triangles, 12 pentagons) & 60 & 30 \\
\hline Truncated Dodecahedron & 32 (20 triangles, 12 decagons) & 90 & 60 \\
\hline Truncated Icosahedron & 32 (12 pentagons, 20 hexagons) & 90 & 60 \\
\hline Rhombicosidodecahedron & 62 (20 triangles, 30 squares, 12 pentagons) & 120 & 60 \\
\hline Truncated Icosidodecahedron & 62 (30 squares, 20 hexagons, 12 decagons) & 180 & 120 \\
\hline Snub Dodecahedron & 92 (80 triangles, 12 pentagons) & 150 & 60 \\
\hline
\end{tabular}

Table 4. For the model, shown in Fig. 1, the vertices of a rhombicuboctahedron

\begin{tabular}{|c|c|c|c|}
\hline corner & $\mathrm{x}$ & $\mathrm{y}$ & $\mathrm{z}$ \\
\hline 1 & $\mathrm{a}$ & $-\mathrm{a}$ & 1,85 \\
\hline 2 & $\mathrm{a}$ & $\mathrm{a}$ & 1,85 \\
\hline 3 & $-\mathrm{a}$ & $\mathrm{a}$ & 1,85 \\
\hline 4 & $-\mathrm{a}$ & $-\mathrm{a}$ & 1,85 \\
\hline 5 & $\mathrm{a}$ & $-1,85$ & $\mathrm{a}$ \\
\hline 6 & 1,85 & $-\mathrm{a}$ & $\mathrm{a}$ \\
\hline 7 & $\mathrm{a}$ & $-1,85$ & $-\mathrm{a}$ \\
\hline 8 & 1,85 & $-\mathrm{a}$ & $-\mathrm{a}$ \\
\hline 9 & $-\mathrm{a}$ & 1,85 & $\mathrm{a}$ \\
\hline 10 & $-1,85$ & $\mathrm{a}$ & $\mathrm{a}$ \\
\hline 11 & $-\mathrm{a}$ & 1,85 & $-\mathrm{a}$ \\
\hline 12 & $-1,85$ & $\mathrm{a}$ & $-\mathrm{a}$ \\
\hline 13 & 1,85 & $\mathrm{a}$ & $\mathrm{a}$ \\
\hline 14 & $\mathrm{a}$ & 1,85 & $\mathrm{a}$ \\
\hline 15 & 1,85 & $\mathrm{a}$ & $-\mathrm{a}$ \\
\hline 16 & $\mathrm{a}$ & 1,85 & $-\mathrm{a}$ \\
\hline 17 & $-1,85$ & $-\mathrm{a}$ & $\mathrm{a}$ \\
\hline 18 & $-\mathrm{a}$ & $-1,85$ & $\mathrm{a}$ \\
\hline 19 & $-1,85$ & $-\mathrm{a}$ & $-\mathrm{a}$ \\
\hline 20 & $-\mathrm{a}$ & $-1,85$ & $-\mathrm{a}$ \\
\hline 21 & $\mathrm{a}$ & $-\mathrm{a}$ & $-1,85$ \\
\hline 22 & $\mathrm{a}$ & $\mathrm{a}$ & $-1,85$ \\
\hline 23 & $-\mathrm{a}$ & $\mathrm{a}$ & $-1,85$ \\
\hline 24 & $-\mathrm{a}$ & $-\mathrm{a}$ & $-1,85$ \\
\hline & & & \\
\hline
\end{tabular}


A rhombicuboctahedron has twenty four-vertices. The vertices of a dodecahedron, whose origin was chosen at the centre of body, are indexed as cartesian coordinates in Table 3.

Assuming the distances of midpoints of all the edges to the origin of the rhombicuboctahedron are normalized. We suppose that edge lenght is $2 \mathrm{a}$.

Now, for example, from eqs.(12), (13) and (21), the C2 rotation of 13th vertex around the z-axis is

$\mathrm{R}^{\prime}=\mathrm{A}_{\mathrm{z}} \mathrm{R}_{13} \mathrm{a}_{\mathrm{z}}{ }^{*}$

$=$

$$
\begin{aligned}
& {\left[\begin{array}{cccc}
0 & 0 & 0 & -1 \\
0 & 0 & -1 & 0 \\
0 & 1 & 0 & 0 \\
1 & 0 & 0 & 0
\end{array}\right]\left[\begin{array}{lrrr}
0 & -1,85 & -\mathrm{a} & -\mathrm{a} \\
1,85 & 0 & -\mathrm{a} & -\mathrm{a} \\
\mathrm{a} & \mathrm{a} & 0 & -1,85 \\
\mathrm{a} & \mathrm{a} & 1,85 & 0
\end{array}\right]\left[\begin{array}{l}
0 \\
0 \\
0 \\
-1
\end{array}\right]} \\
& =\left[\begin{array}{c}
0 \\
-1,85 \\
-\mathrm{a} \\
\mathrm{a}
\end{array}\right]
\end{aligned}
$$

where $\mathbf{A}_{\mathrm{z}}, \mathrm{R}_{13}$ and $\mathrm{a}_{\mathrm{z}}{ }^{*}$ are the (4x4)-matrix representations of rotation $\mathrm{z}$-axis and 13th vertex, $(4 \times 1)$ column matrix representation of the $(4 \times 4)$ matrix of $\mathrm{A}_{\mathrm{z}}{ }^{*}$ respectively. This result of the matrix operation defines the 17 th vertex of the rhombicuboctahedron.

This method is the conventional geometrical method of the rotation operation for rhombicuboctahedron. Using quaternions, the same calculation can be obtained as follows:

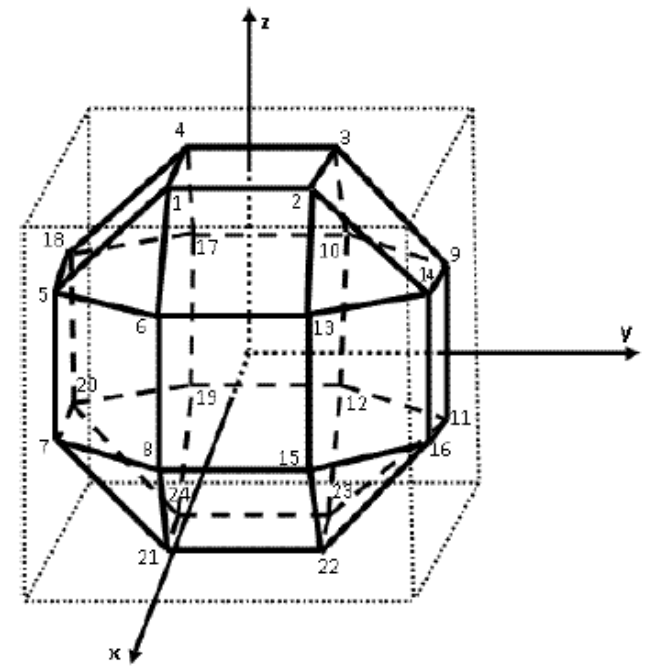

Figure 3. Rhombicuboctahedron

$\mathrm{R}^{\prime}=\mathrm{a}_{\mathrm{z}} \mathrm{R}_{13} \mathrm{a}_{\mathrm{z}}{ }^{*}$

$=\left(1 \mathrm{e}_{12}\right)\left(\mathbf{1}, \mathbf{8 5} \mathbf{e}_{1}+\mathbf{a e}_{2}+\mathbf{a} \mathbf{e}_{3}\right)\left(-\mathrm{e}_{12}\right)$

$=\left(-1,85 \mathrm{e}_{1}+-\mathrm{ae}_{2}+\mathrm{ae}_{3}\right)$

This result is equal to the quaternionic definition of 17th vertex, as well. Now we investigate another rotation operation of 17 th $\mathrm{z}$-axis around the straight line. 
The rotation $90^{\circ}$ of 17 th vertex z-axis around this straight line.

$\mathrm{R}^{\prime}=\mathrm{a}_{\mathrm{z}} \mathrm{R}_{17} \mathrm{a}_{\mathrm{z}}^{*}$

$=\left(\frac{\sqrt{2}}{2}+\frac{\sqrt{2}}{2} \mathrm{e}_{12}\right)\left(-1,85 \mathrm{e}_{1}-\mathrm{ae}_{2}+\mathrm{ae}_{3}\right)\left(\frac{\sqrt{2}}{2}-\frac{\sqrt{2}}{2} \mathrm{e}_{12}\right)$

$=\left(-\mathrm{ae}_{1}+1,85 \mathrm{e}_{2}+\mathrm{ae}_{3}\right)$.

This result is equal to the quaternionic definition of 9 th vertex. The rotation $45^{\circ}$ of 9 th vertex $z$-axis around this straight line

$\mathrm{R}^{\prime}=\mathrm{a}_{\mathrm{z}} \mathrm{R}_{9} \mathrm{a}_{\mathrm{z}}^{*}$

$=\left(\frac{\sqrt{\sqrt{2}+2}}{2}+\frac{\sqrt{2-\sqrt{2}}}{2} \mathrm{e}_{12}\right)\left(-\mathrm{ae}_{1}+1,85 \mathrm{e}_{2}+\mathrm{ae}_{3}\right)\left(\frac{\sqrt{\sqrt{2}+2}}{2}-\frac{\sqrt{2-\sqrt{2}}}{2} \mathrm{e}_{12}\right)$

$=\left(\mathrm{ae}_{1}+1,85 \mathrm{e}_{2}+\mathrm{ae}_{3}\right)$.

This result is equal to the quaternionic definition of 14th vertex. The reflection operations can be also defined by the Clifford algebra elements. According to the xz-plane, the reflection of 2 th vertex with Clifford algebra is

$\mathrm{R}^{\prime}=\sigma_{\mathrm{xz}} \mathrm{R}_{10} \sigma_{\mathrm{xz}}{ }^{-1}$

$=e_{13}\left(a_{1}+a_{2}+1,85 e_{3}\right) e_{13}$

$=\mathrm{ae}_{1}-\mathrm{ae}_{2}+1,85 \mathrm{e}_{3}$,

where $\sigma_{\mathrm{xz}}{ }^{-1}$ is the inverse of $\sigma_{\mathrm{xz}}$ operation. The reflection of 10th vertex on the xz-plane is equal to the 1 th vertex. After this reflection of the 2 th vertex on the xz-plane, the reflection of 1 th vertex on the xyplane can be written as

$\mathrm{R}^{\prime \prime}=\sigma_{\mathrm{xy}} \mathrm{R}^{\prime} \sigma_{\mathrm{xy}}{ }^{-1}$

$=\sigma_{\mathrm{xy}}\left(\sigma_{\mathrm{xz}} \mathrm{R}_{2}{\sigma_{\mathrm{xy}}}^{-1}\right) \sigma_{\mathrm{xy}}{ }^{-1}$

$=\left(\sigma_{\mathrm{xy}} \sigma_{\mathrm{xz}}\right) \mathrm{R}_{2}\left(\sigma_{\mathrm{xz}} \sigma_{\mathrm{xy}}\right)$

$=\mathrm{ae}_{1}-\mathrm{ae}_{2}-1,85 \mathrm{e}_{3}$.

This result is equal to the quaternionic definition of 21 th vertex in Table 4.

The rotations $45^{\circ}\left(\mathrm{C}_{8}\right), 90^{\circ}\left(\mathrm{C}_{4}\right), 180^{\circ}\left(\mathrm{C}_{2}\right)$ of around axis which are perpendicular to the this surface, from the midpoint of each square face and the rotations $60^{\circ}\left(\mathrm{C}_{6}\right)$ of around axis which are perpendicular to the this surface, from the midpoint of each face of triangle are possible. 3 reflection in a plane perpendicular to a 3 -fold axis and the rotoreflections $180^{\circ}\left(\mathrm{S}_{2}\right)$ of around the axis drawn from each corner to the other corner are also possible. $\mathrm{O}_{\mathrm{h}}(* 432)$ group; achiral octahedral symmetry or full octahedral symmetry; is made up of all this operations of rotation and reflection.

\section{CLASSICS PARTICLES' ENERGY AT ARCHIMEDEAN SOLIDS WITH CLIFFORD ALGEBRA}

The reflection and rotation operations in the solid state physics and molecular physics play an important role. The symmetry operations can be easily applied on the regular polyhedra, which are called Platonic Solids. At the same time the operations can be apply to The Archimedean solids. 
Any point represented by $\mathbf{R}$ quaternion transforms to the a new quaternionic point at the end of a rotation defined by $\mathbf{A}$ unit quaternion. This new quaternion is

$\mathbf{R}^{\prime}=\mathbf{A} \mathbf{R} \mathbf{A}^{*}$,

where $\mathbf{A} *$ is complex conjugate of $\mathbf{A}$.

The reflection of $\mathbf{r}$ across the line a, namely the mirror image $\mathbf{r}$ ' of $\mathbf{r}$ with respect to $\mathbf{a}$, is given by $\mathbf{r}^{\prime}=$ ara $^{-1}$

For example rhombicuboctahedron's $17^{\text {th }}$ vertices's replasement and potential energy with Clifford algebra:

$\mathrm{R}^{\prime}=\mathrm{a}_{\mathrm{z}} \mathrm{R}_{17} \mathrm{a}_{\mathrm{z}}^{*}$

$=\left(\frac{\sqrt{2}}{2}+\frac{\sqrt{2}}{2} \mathrm{e}_{12}\right)\left(-1,85 \mathrm{e}_{1}-\mathrm{ae}_{2}+\mathrm{ae}_{3}\right)\left(\frac{\sqrt{2}}{2}-\frac{\sqrt{2}}{2} \mathrm{e}_{12}\right)$

$=\left(-\mathrm{ae}_{1}+1,85 \mathrm{e}_{2}+\mathrm{ae}_{3}\right)$.

This result is equal to the quaternionic definition of 14 th vertex.

We can calculate difference of potential energy for this two points. The potential energy of this points are equal.

14 th vertex's coordiante is $\left(a_{1}+1,85 e_{2}+a_{3}\right)$ and 21 th vertex's coordiante is ae $e_{1-} a_{2}-1,85 e_{3}$.

$\mathrm{R}_{21}{ }^{\prime}-\mathrm{R}_{14}=\left(\mathrm{ae}_{3}\right)-\left(-1,85 \mathrm{e}_{3}.\right)=2,85 \mathrm{e}_{3}$

The difference of potential energy for this points is

$\mathrm{U}=\mathrm{mg}(2,85)$.

Table 5. Potential energies of rhombicuboctahedron's edges

( $\mathrm{m}$ is mass, $\mathrm{g}$ is gravitational acceleration)

\begin{tabular}{|l|c|}
\hline $\begin{array}{c}\text { Vertices } \\
\text { number's }\end{array}$ & z \\
\hline 1 & 1,85 gh \\
\hline 2 & 1,85 gh \\
\hline 3 & 1,85 gh \\
\hline 4 & 1,85 gh \\
\hline 5 & a gh \\
\hline 6 & a gh \\
\hline 7 & -a gh \\
\hline 8 & -a gh \\
\hline 9 & a gh \\
\hline 10 & a gh \\
\hline 11 & -a gh \\
\hline 12 & - a gh \\
\hline 13 & a gh \\
\hline 14 & a gh \\
\hline 15 & -a gh \\
\hline 16 & -a gh \\
\hline 17 & a gh \\
\hline 18 & a gh \\
\hline 19 & -a gh \\
\hline 20 & -a gh \\
\hline 21 & $-1,85$ gh \\
\hline 22 & $-1,85$ gh \\
\hline 23 & $-1,85$ gh \\
\hline 24 & $-1,85$ gh \\
\hline & \\
\hline
\end{tabular}




\section{CONCLUSIONS}

In this study we apply Clifford Algebra to Archimedean Solids. They are semi-symmetric solids. The geometrical methods and matrices are used for the investigation of symmetry operations of the symmetric solid too [5]. Clifford algebras are algebras of geometries and quaternions are hypercomplex numbers [15]. In this study, Clifford algebra and quaternions are used for the symmetry operations. When these operations are made with Clifford algebra and quaternions, it is obvious that the calculations are easy and compact. The quaternions and Clifford algebra are much simpler to apply to the symmetry operations than the conventional methods of molecular symmetry. This method can be applied to the more complex structures.

\section{REFERENCES}

[1] Doran C, Lasenby A. Geometric Algebra for Physicists, Cambridge University Press, 2003.

[2] KlineM , Mathematical Though from Ancient to Modern Times. Oxford University Press, 1972.

[3] W. K. Clifford, Am. J. Math. 1350., 1878.

[4] Altmann S L. Rotations, quaternions, and double groups. Clarendon Press, Oxford, 1994.

[5] Kilic A. Ozdas K. Tanisli M. An Investigation of Symmetry Operations with Clifford Algebra 54(3) 2004.

[6] Girard PR, Quaternions, Clifford Algebra and Relativistic Physics, Birkhouser, 2007.

[7] Funda J, Paul RP, A Comparison of Transforms and Quaternions in Physics, Proc. of 1988 IEEE Int. Conference on Robotics and Automation, Philadelphia, p.886,1988.

[8] Tanışlı M, Acta Physica Slovaca, 53(3) (2003) 253., 2012.

[9] Rodriques O, Journal de Mathematiques Pures et Appliquees 5, 380, 1840.

[10] Lounesto P, Lectures on Clifford Geometric Algebras, TTU Press, Cookeville, TN, USA, 2002.

[11] Jancewicz B., Multivectors and Clifford Algebra in Electrodynamics, World Scientific, 1989.

[12] J. Snygg: Clifford Algebra, Hardcover, 1997.

[13] Pokorny A, Herzig P, Altman SL, Spectrochimica Acta, A 57 1931, 2001.

[14] Williams R, The Geometrical Foundation of Natural Structure, Paperback, 1979.

[15] Anderson S, JR. Joshi GC., Physics Essays, 6, 308, 1993. 